\title{
Fluorescence spectroscopy of normal and follicular cancer samples from human thyroid
}

\author{
G. Giubileo a, ${ }^{\text {a, }}$ F. Colao ${ }^{\text {a }}$, A. Puiu ${ }^{\text {b }}$, G. Panzironi ${ }^{\text {c }}$, F. Brizzi ${ }^{\text {a }}$ and P. Rocchini ${ }^{\text {a }}$ \\ ${ }^{a}$ ENEA, Laboratorio di Spettroscopia Molecolare, Via E. Fermi 45, 00044-Frascati, Italy \\ ${ }^{\mathrm{b}}$ NILPRP, Magurele-Bucharest, Laser Department, Romania \\ c Università "La Sapienza”, III Clinica Chirurgica, Roma, Italy
}

\begin{abstract}
An autofluorescence analysis has been performed on healthy as well as tumour thyroid tissue samples to distinguish follicular cancer from normal thyroid. Complete spectra and synchronous spectra have been recordered from properly stored samples. Fluorescence bands located at $350 \mathrm{~nm}$ and $400 \mathrm{~nm}$ has been observed in the analysed cancer samples.
\end{abstract}

Keywords: Autofluorescence, follicular thyroid cancer, cancer diagnosis, FNA biopsy

\section{Introduction}

\subsection{Thyroid follicular cancer}

The thyroid is a small gland in the front of the neck just under the larynx. It is also called the activity gland because it produces two main hormons, tri-iodothyronine and the thyroxine, which are needed to keep the body functioning at its normal rate. The thyroid gland needs a regular supply of iodine in order to produce thyroxine. Thyroid may develop cancer pathology; there are four types of cancer of the thyroid: papillary, follicular, medullary, anaplastic. Follicular cancer generally comprises about $25 \%$ of all diagnosed thyroid cancers and is usually the most aggressive form among other common types. It is most often discovered as a painless lump in the thyroid, usually occurs after the age of 40, preferentially in females with respect to males. Unlike other types of thyroid cancer, it can be difficult to diagnose the follicular cancer without performing surgery.

A fine needle aspiration (FNA) biopsy, the most common resolutive diagnosing procedure, often cannot distinguish between the follicular adenoma (the follicular cancer precursor), or the follicular cancer and a completely benign condition called nontoxic nodular goiter. Even a coarse needle biopsy, which is tipically more accurate than an FNA, cannot always provide an answer since it is only able to distingiush a follicular neoplasm (which include both adenoma and cancer) versus nontoxic nodular gotier in about $40 \%$ of the cases. This difficulty in diagnosis is one of the most frustrating topics for physicians involved in the thyroid study, because it means that surgery is often the only way of definitively diagnosing a thyroid nodule, determining unnecessary damages to a number of patients $[5,6,8]$. Consequently any new method allowing a more accurate diagnosis of the follicular cancer would be welcome.

\footnotetext{
*Corresponding author. Tel.: +39 06 94005768; E-mail: giubileo@frascati.enea.it.
} 


\subsection{Fluorescence analysis}

The spectral analysis of induced fluorescence could provide a very simple and accurate diagnostic method. Many efforts have already been made to put the spectral analysis of induced fluorescence at work to distinguish normal tissue from tumour tissue in different regions of the human body [1-3, $7,9-11]$. The idea of using the tissue fluorescence to diagnose diseases was suggested 50 years ago as an useful method to help the surgeon by putting in evidence the tumour tissue during operations. Nowadays, also due to the increasing development of the laser sources, the tissue fluorescence analysis can be performed more efficiently, even in endoscopic procedures.

Intrinsic fluorescence (autofluorescence) from tissue is caused by the presence of fluorescing molecular compounds in the biomolecules constituting the cells. Changes in the autofluorescence spectra coming from normal and cancer tissue are likely due to changes in the molecular composition which determine changes in the spectral response. The autofluorescence analysis is non-invasive and does not produce negative side effects, differently from the hazardous technique based on the fluorescence coming from tumour localizing agents (like aminolevulinic acid) injected into the body and retained by the tissue for a period, and in the meanwhile activated by radiation at a given wavelength.

The proposal of a diagnosis protocol of thyroid tumour based on the autofluorescence analysis would be desirable for different reasons. Firstly, as a good help in identifying the follicular thyroid tumours which elude the usual cytological assay of the biopsies obtained by the fine needle aspiration (FNA) technique. Secondly, it would be good to improve the diagnostic protocol of thyroid pathology making the job of the pathologist easier and time saving, as well as error free.

\subsection{Purpose of this work}

With the above depicted scenario in mind we started a feasibility study to look for any analytical feature in the spectral response of different human thyroid samples useful to set up a fluorescence analysis protocol for distinguishing follicular from normal thyroid tissue and eventually realize a dedicated easy to use laser induced fluorescence (LIF) apparatus for the real time diagnosis of thyroid follicular cancer during an FNA session. The study has been performed on samples of follicular cancer and compared with samples of healthy tissue belonging to the same human thyroid coming from surgial biopsies already decided as necessary by the clinical staff on the patients involved. The experimental work has been performed at ENEA Molecular Spectroscopy Laboratory in Frascati on samples provided by the III Clinica Chirurgica at La Sapienza University in Rome.

\section{Materials and methods}

\subsection{Spectroscopy}

The fluorescence spectra were produced by a PTI Quanta Master spectrafluorometer equipped by a double monochromator $(0.2 \mathrm{~m}$ length and 300 grate/mm grating in Czerny-Turner configuration) allowing the selection of both excitation and emission wavelengths. A schematic of the experimental set-up is given in the Fig. 1. The excitation radiation was provided by a Xe arc lamp (Osram 630, $75 \mathrm{~W}$ ) emitting in the UV-visible range from $190 \mathrm{~nm}$ to $1100 \mathrm{~nm}$. The apparatus has a nominal $1.25 \mathrm{~nm}$ spectral resolution, but a $5 \mathrm{~nm}$ bandwidth has been used for both excitation and emission, coming out from a compromise between sensitivity and spectral resolution. The system operation and data acquisition are 


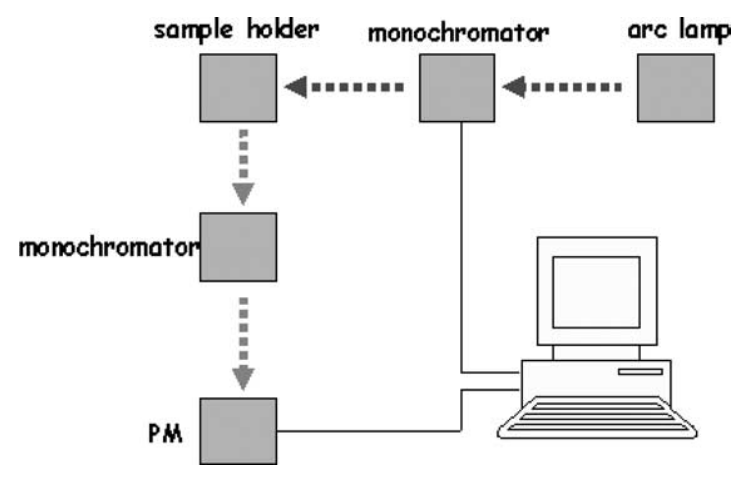

Fig. 1. Schematic of the experimental layout.

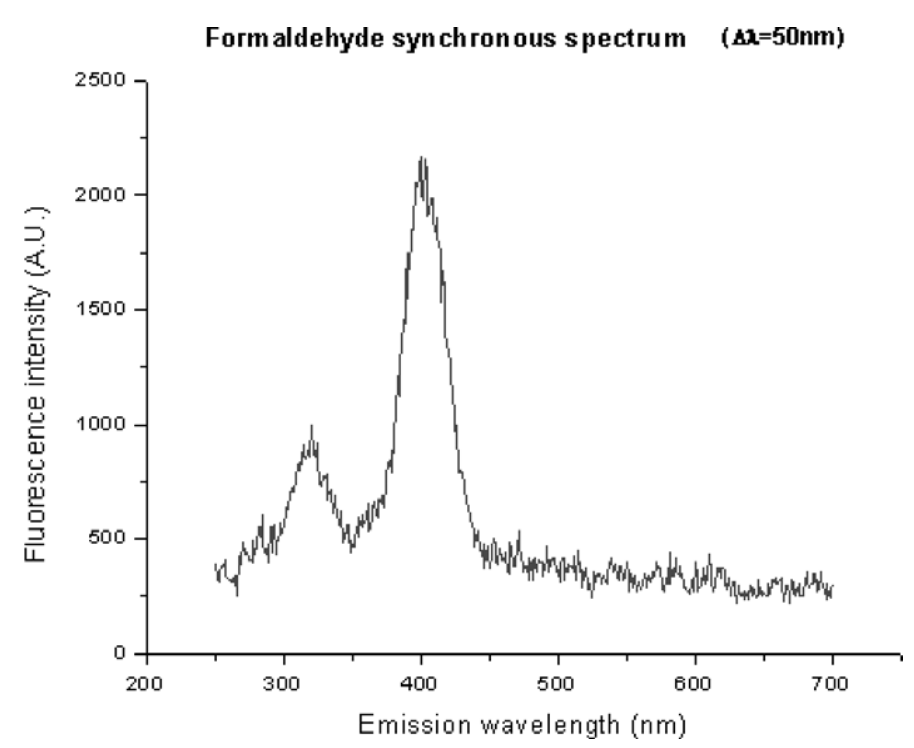

Fig. 2. Typical emission spectrum from formaldehyde.

under the control of a Personal Computer. Felix ${ }^{\mathrm{TM}}$ software operating under Windows OS was used to control the spectrafluorometer and to acquire data, while Surfer ${ }^{\mathrm{TM}}$ software was used for spectra visualisation. Emission, excitation and synchronous spectra can be performed. Emission spectra are acquired by keeping the excitation wavelength constant, while excitation spectra require the emission wavelength to be constant; finally synchronous spectra were acquired by keeping constant the difference of emission and excitation wavelength. Typical emission and synchronous spectra from PTI QM are reported in Fig. 2 and Fig. 3, respectively. Here the Raman emission of storing medium can be easily recognised, while we note the very low emission in the spectral region around $400 \mathrm{~nm}$. Actually when the emission monochromator is tuned to a frequency which is exactly an integer multiple of the excitation wavelength, a very intense spike in the emission spectra is detected. This spike is due to the second order grate transmission and its presence can be avoided by acquiring the emission spectra in two sections, the first from $\lambda_{\mathrm{ex}}+\Delta \lambda$ to $2 \lambda_{\mathrm{ex}}-\Delta \lambda$ and the second from $2 \lambda_{\mathrm{ex}}+\Delta \lambda$ to $\lambda_{\max }$. Complete spectral characterisation in a form of $2 \mathrm{D}$ graph can be obtained by combining fluorescent emission spectra with excitation wavelengths ranging from 200 to $700 \mathrm{~nm}$. Each point in the XY plane can be considered as 


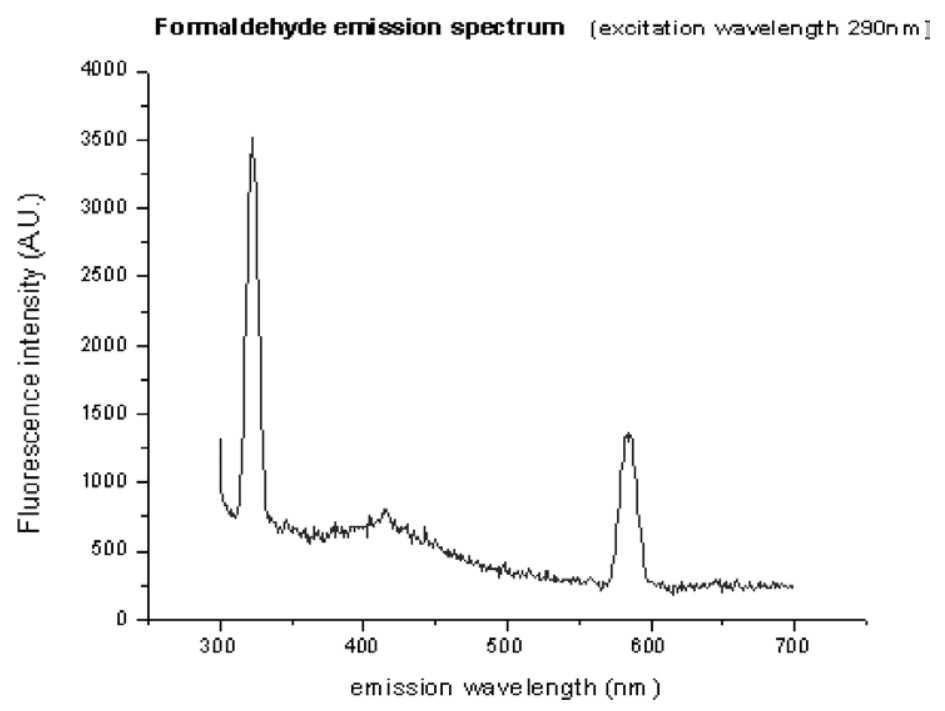

Fig. 3. Typical synchronous spectrum from formaldehyde.

made up by a couple of excitation and emission wavelength values; a relative fluorescence intensity is then associated to a grey level colour in a logarithmic scale. This combined spectrum contains the most complete fluorescence analysis obtainable by our spectrofluorometer. Actually 50 emission spectra are acquired in the spectral region varying $\lambda_{\text {ex }}$ from 200 to $700 \mathrm{~nm}$ in step of $\Delta \lambda_{\mathrm{ex}}=10 \mathrm{~nm}$. Although a long time is needed to collect a so large amount of spectral information, advantages are gained because once spectral data have been recorded, all kind of usual spectra can be derived. An emission spectrum at an arbitrary $\lambda_{\text {ex }}$ wavelength can be obtained by crossing the 2D spectrum along vertical line, i.e. by keeping the excitation wavelength fixed; crossing the spectrum with an horizontal line we get an excitation spectra; synchronous spectra can be obtained crossing the 2D spectrum with positive slanted lines; finally, isoenergetic spectrum can be obtained along equilateral hyperbolas with vertical asymptote.

\subsection{Samples preparation}

Convenient parts of human thyroids to be analysed have been prelevated from patients after necessary surgical biopsies and put into formaldehyde for storing purposes as well as for transportation from the Hospital to the ENEA Laboratories. Formaldehyde was already demonstrated to be the most convenient medium compared with ethyl alcohol and paraffin [4]. Parts of both cancer and healthy tissue were separated from the same excised thyroid to allow a comparison between the spectral features of the two kinds of tissue. Samples as thin as $200 \mu \mathrm{m}$ were cut from those pieces and were put between two silica quartz slabs; the entire arrangement was placed in the sample holder of the spectrafluorometer. Complete induced fluorescence spectra were then recorded and subsequently elaborated to extract the information.

\section{Results}

\section{1. $2 D$ spectra}

In Fig. 4 the complete 2D spectrum of a healthy thyroid tissue is given. It is not evident the presence of any structured spectral area, neither any emission/excitation spectral band determined by specific chro- 


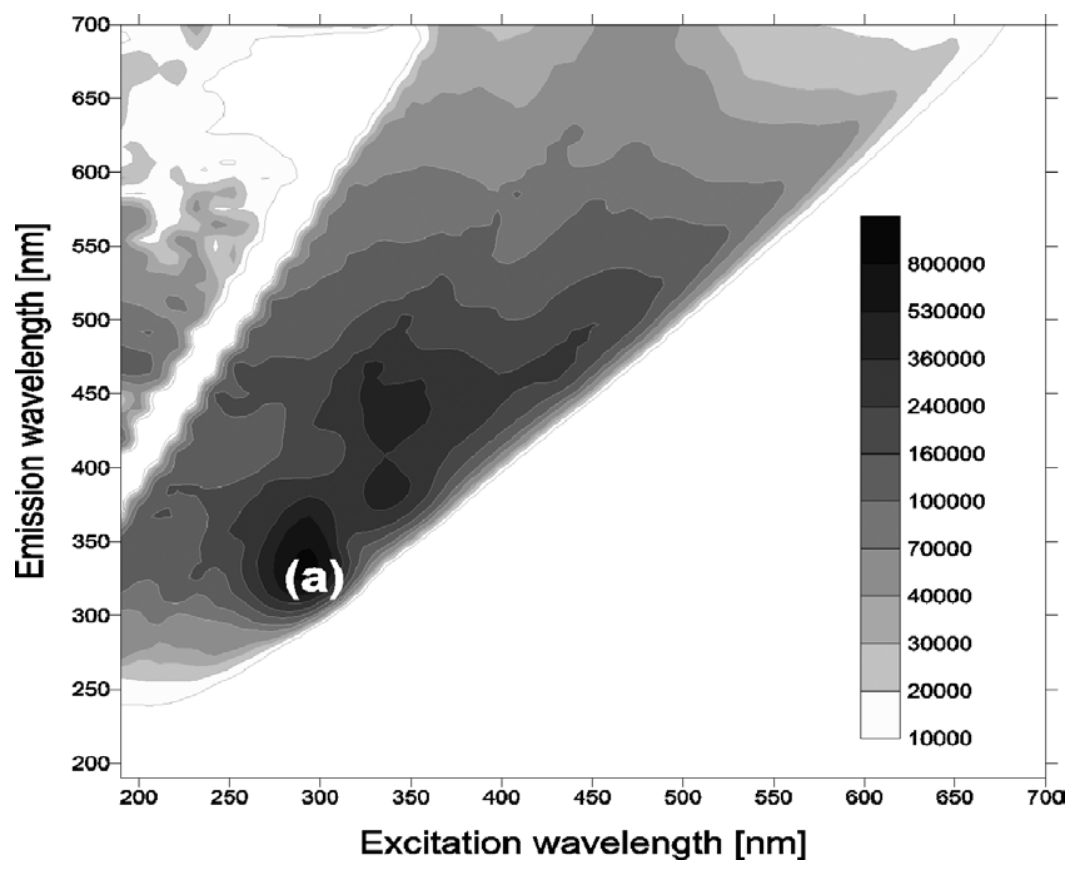

Fig. 4. Healthy tissue 2D spectrum.

mophors; instead we can observe a broad emission peak. A slight tendency to form undefined bands having a tail in direction of the higher values of wavelength can be observed. Regarding the strongest emissions, an intense fluorescence band, named as (a) in Fig. 4, excited at around $300 \mathrm{~nm}$ is observable inside a moderately intense fluorescence area distributed between $300 \mathrm{~nm}$ and $450 \mathrm{~nm}$, with $30 \mathrm{~nm}$ spectral width in excitation and $90 \mathrm{~nm}$ spectral width in emission. No Raman structure is easily recognisable in this spectrum.

Similar features can be recognised while observing the results from a tumour tissue. The complete 2D spectrum of a follicular cancer tissue of the same patient, given in Fig. 5, has been compared with the healthy tissue 2D spectrum of Fig. 4. Beside the same fluorescence band showed by the healthy tissue, a second broaden band named as (b) excited at $350 \mathrm{~nm}$ (100 nm times $140 \mathrm{~nm}$ spectral width) appears in this spectrum, both of them emerging from a less intense fluorescence background distributed between $300 \mathrm{~nm}$ and $450 \mathrm{~nm}$. Similarly to the spectrum of Fig. 4, no Raman structure is visible in Fig. 5 spectrum. Apart for the presence of the second fluorescence band, the general features of two spectra seem to be quite similar.

The comparison among different fluorescence spectra will be made by taking into account the overall spectrum appearance, i.e. by identifying the spectral emission in terms of their presence, their position, width and relative intensity. The spectral shape does not depend on sample orientation with respect to the direction of the excitation light beam, thus allowing for unambiguous comparison of spectra taken at different times and with slight different geometrical position due to imperfect sample repositioning or nonconstant sample size. On the other hand the overall spectrum intensity from different samples has not to compare because it is strongly dependent on poor controlled experimental settings like sample size, sample position, long term excitation intensity variation, long term drift of the detection unit sensitivity. 


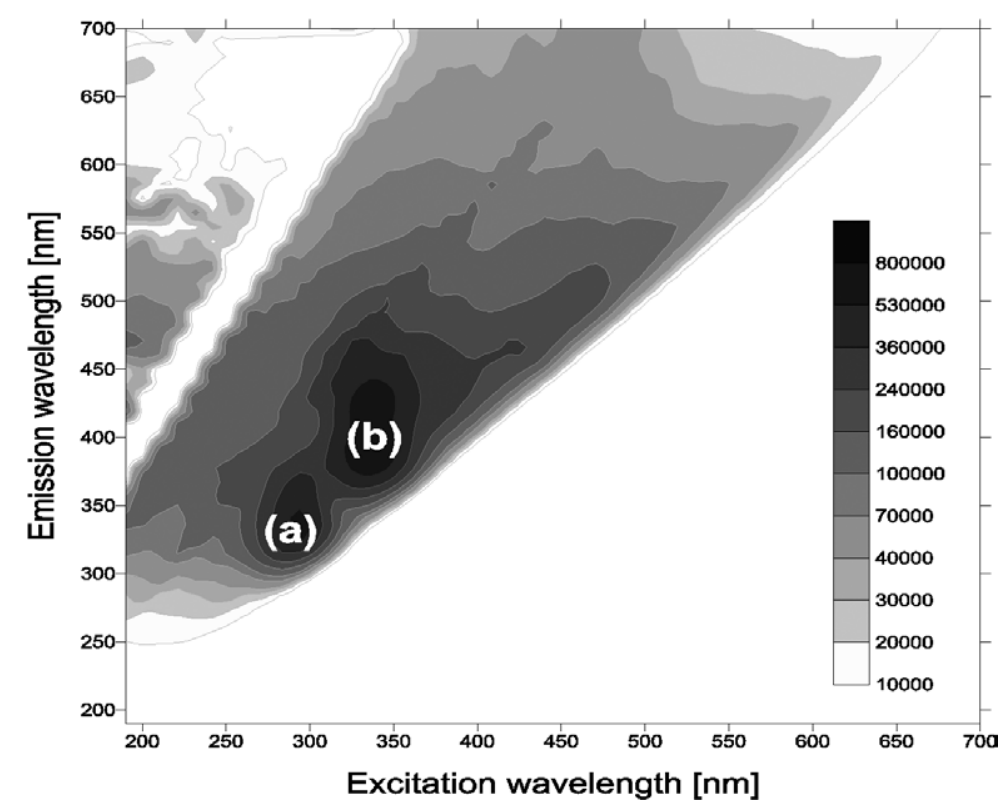

Fig. 5. Tumour tissue 2D spectrum.

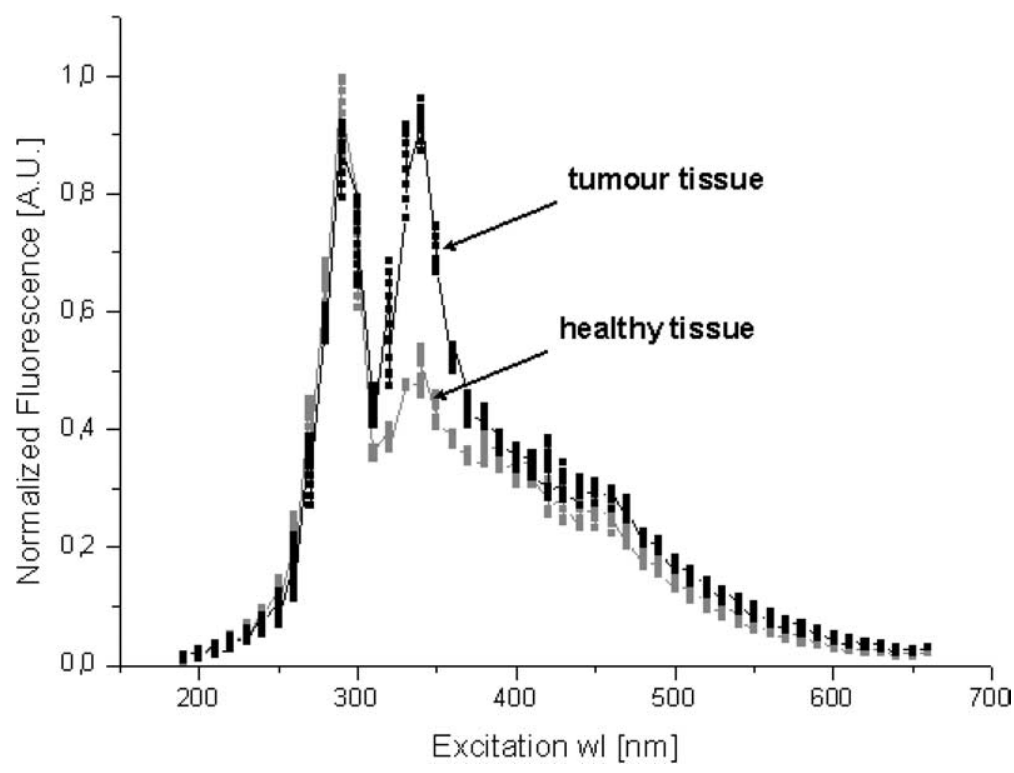

Fig. 6. Synchronous spectra at $\Delta \lambda=50 \mathrm{~nm}$ from normal tissue and from tumour tissue extracted by interpolation from the corresponding 2D spectra.

\subsection{Synchronous spectra}

The relation among the two useful spectral areas could be put in evidence by crossing the 2D spectrum along a 45 degrees tilted line. This means to build up a graph by fluorescence data in which the difference $\Delta \lambda$ between the emission wavelength $\lambda_{\mathrm{em}}$ and excitation wavelength $\lambda_{\mathrm{ex}}$ is kept constant (see Section 2). In Fig. 6 two such synchronous spectra at $\Delta \lambda=50 \mathrm{~nm}$ have been reported, by interpolation 


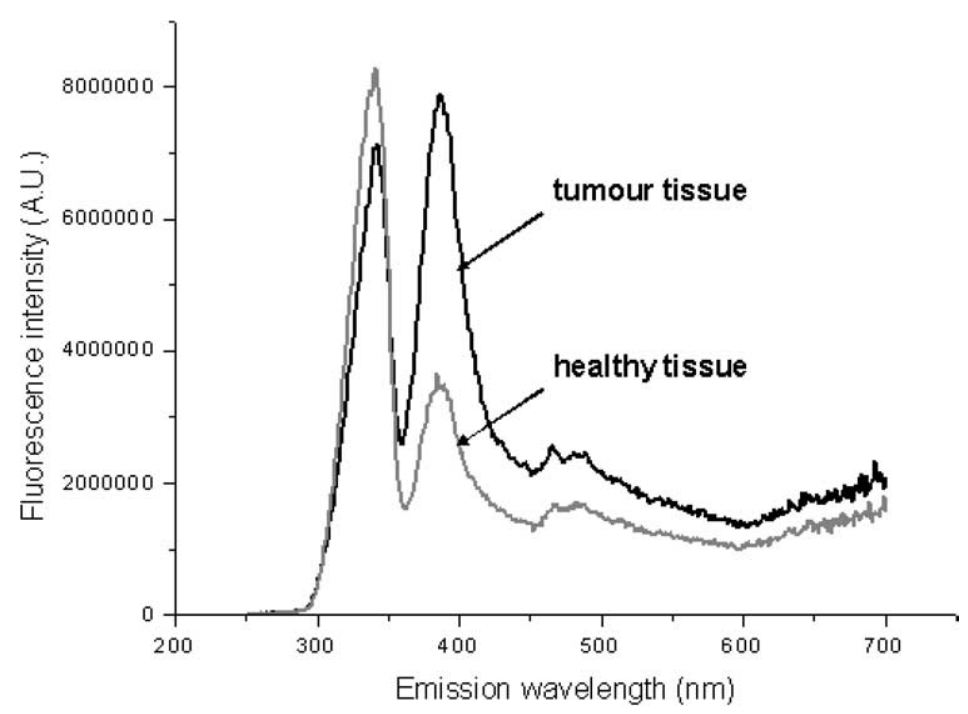

Fig. 7. Synchronous spectra at $\Delta \lambda=50 \mathrm{~nm}$ from normal tissue and from tumour tissue directly recorded from the tissue samples.

data corresponding the 2D spectrum respectively from healthy tissue shown in Fig. 4 and from tumour tissue shown in Fig. 5. They have been reported on the same graph to allow a direct comparison between them. The same synchronous spectra at $\Delta \lambda=50 \mathrm{~nm}$ have also been directly recorded from the tissue samples and reported in Fig. 7. Note that the storing medium (see Fig. 3) has a very large difference with thyroid samples, thus allowing unambiguous identification of spectral features in case of biological samples.

\section{Discussion}

Apart from a generic overall higher intensity of the tumour spectrum with respect to the normal one, the observed fluorescence bands do not appear very specific of the tumour tissue, and the single scan spectra (excitation or emission) cannot make it recognisable with respect to the healthy one. On the other hand, the type of tissue could be identified through a simultaneous comparison of the two bands. Unfortunately the two fluorescence bands can be seen on emission (respectively excitation) spectra only one at a time, but not simultaneously on the same spectrum, because they belong to two not overlapping spectral areas which result well separated when crossing the 2D spectrum along a vertical or horizontal line. In other words, if we take into account a single emission spectrum with the excitation at $300 \mathrm{~nm}$ (or a single excitation spectrum with emission kept at $340 \mathrm{~nm}$ ) we record only the (a) band without the (b) band; in the same way, if we perform a single emission spectrum with the excitation at $350 \mathrm{~nm}$ (or a single excitation spectrum with the emission kept at $390 \mathrm{~nm}$ ) we record only the (b) band without the (a) band. Consequently, it is not possible a direct comparison between the two structures on such a kind of graph. The graphs of Fig. 6 and Fig. 7 show clearly the advantages of synchronous spectrum representation because it includes both the (a) and the (b) bands, now appearing very well spectrally separated. So in our case it is more fruitful to analyse the thyroid tissue by means of synchronous spectra at the selected wavelength instead of using couples of emission/excitation spectra. 


\subsection{Spectral indicator}

As it can be seen, a difference between the healthy tissue and the tumour tissue is well evident, in comparing the intensity of the (b) peak to the intensity of the (a) peak, the latter assumed as reference. In the graphs reported in Fig. 7 the spectral ratio $R=F_{a} / F_{b}$ of the fluorescence intensity excited at $290 \mathrm{~nm}$ and emitted at $340 \mathrm{~nm}\left(F_{a}\right)$ to the fluorescence intensity excited at $340 \mathrm{~nm}$ and emitted at $390 \mathrm{~nm}\left(F_{b}\right)$ can be defined. The dimensionless spectral ratio $R$, proposed as tissue indicator, assumes small values for healthy tissues while high values are indicative for the presence of a tumour. In the reported study we found the following values:

\begin{tabular}{ccc}
\hline Parameter & Healthy tissue & Tumour tissue \\
\hline$R$ & $2.3 \pm 4 \%$ & $0.9 \pm 4 \%$ \\
\hline
\end{tabular}

The percentage error as high as $4 \%$ affecting the $R$ parameter came out from a statistical analysis performed on measurements repeated 100 times on the same sample by keeping constant the experimental conditions. As above proposed, relatively to the analysed case it is possible to distinguish the healthy tissue from the tumour tissue. The reported results obtained from the analysed study case are encouraging, but they have to be considered just as a feasibility study whose purpose was to identify a convenient analysis protocol to be further confirmed and validated by additional clinical studies. The outlined method has been applied to the analysis of a number of different samples. Preliminary results confirm the trend found in our study, although a more accurate statistical analysis is still required to properly assess both the natural variability in samples coming from one patient (different samples from the same thyroid) as well as in samples of healthy and tumour thyroids from different patients.

\section{Conclusions}

Due to the poor number of investigated samples, the feasibility study reported in this paper does not yet possess an immediate diagnosis value, but it has a great value in defining the instrument setting. In fact we were looking for the better measurement conditions useful for enhancing the analytical spectral structures and their evaluation in a measurement easier and faster than a complete 2D spectrum study. Finally, we can conclude that:

(1) The best results have been obtained by analysing synchronous spectra more than excitation or emission spectra.

(2) The dimensionless spectral ratio $R=F_{a} / F_{b}$ can be used as tissue indicator in this case. $F_{a}$ is defined as the fluorescence intensity excited at $290 \mathrm{~nm}$ and emitted at $340 \mathrm{~nm}$, while $F_{b}$ is defined as the fluorescence intensity excited at $340 \mathrm{~nm}$ and emitted at $390 \mathrm{~nm}$.

(3) Low values for $R$ could be related to the thyroid follicular cancer tissue, while high $R$ values are related to the healthy thyroid tissue.

\section{References}

[1] I.R. Goellner, H. Gharib, C.S. Grant and D.A. Johnson, Fine needle aspiration cytology of the thyroid, Acta Cytol. 31 (1987), 587-590.

[2] T.L. Hall, L.J. Layfield, A. Phillippe and D.L. Rosenthal, Sources of diagnostic error in fine needle aspiration of the thyroid, Cancer 63 (1989), 718-725. 
[3] L. MacDonald and H.M. Yazdi, Nondiagnostic fine needle aspiration biopsy of the thyroid gland: a diagnostic dilemma, Acta Cytol. 40 (1996), 423-428.

[4] O. A'Amar, D. Lignon, O. Menard, H. Begorre, F. Guillemin and E. Yvroud, Autofluorescence spectroscopy of normal and malignant tissue: both in vivo and ex vivo measurements in the upper aero-digestive tract and lung tissues, SPIE Proceedings 2679 (1996), 42-50.

[5] R.R. Alfano, B. Tata, J. Cordero, P. Tomashefsky, F.W. Longo and M.A. Alfano, Laser induced fluorescence spectroscopy from native cancerous and normal tissue, IEEE Journal of Quantum Electronics 20(12) (1984), 1507-1511.

[6] S. Andersson-Engels, J. Joahnsson, U. Stenram, K. Svanberg and S. Svanberg, Malignant tumor and atherosclerotic plaque diagnosis using laser-induced fluorescence, IEEE Journal of Quantum Electronics 26(12) (1990), 2207-2215.

[7] N. Kollias, G. Zonios and G.N. Stamatas, Fluorescence spectroscopy of skin, Vibrational Spectroscopy 28 (2002), 17-23.

[8] B. Mayinger, P. Horner, M. Jordan, C. Gerlach, T. Horbach, W. Hohenberger and E.G. Hahn, Endoscopic fluorescence spectroscopy in the upper GI tract for the detection of GI cancer: initial experience, The American Journal of Gastroenterology 96(9) (2001), 2616-2621.

[9] M. Sartori, R. Sauerbrey, S. Kubodera, F.K. Tittel, R. Roberts and P.D. Henry, Autofluorescence maps of atherosclerotic human arteries, IEEE Journal of Quantum Electronics 23(10) (1987), 1794-1797.

[10] Y. Yang, A. Katz, E.J. Celmer, M. Zurawska-Szczepaniak and R.R. Alfano, Optical spectroscopy of benign and malignant breast tissue, SPIE Proceedings 2679 (1996), 51-54.

[11] G. Giubileo, F. Colao, P. Rocchini and G. Panzironi, Spectral analysis of induced fluorescence in thyroid tissue, SPIE Proceedings 4252 (2001), 132-139. 


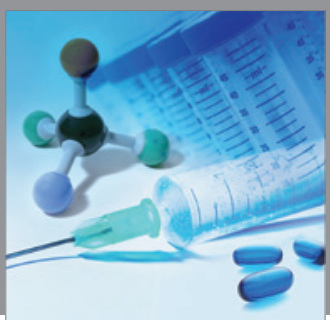

International Journal of

Medicinal Chemistry

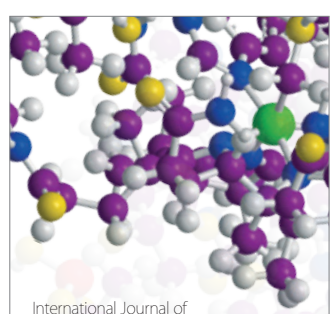

Carbohydrate Chemistry

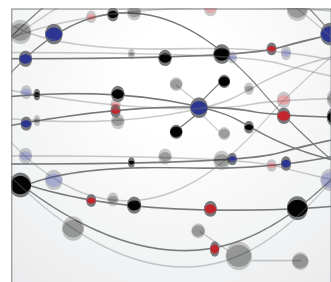

The Scientific World Journal
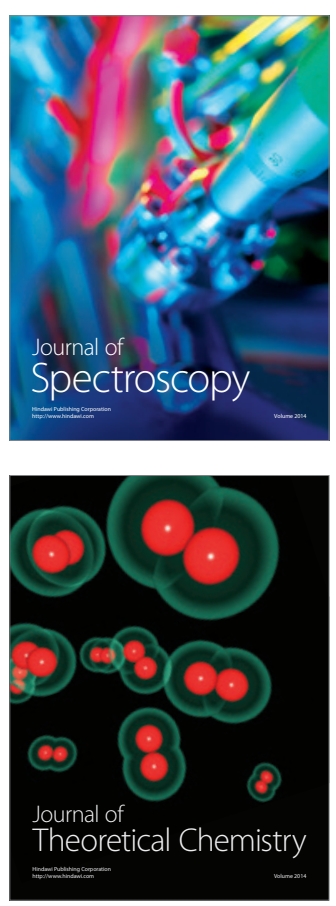
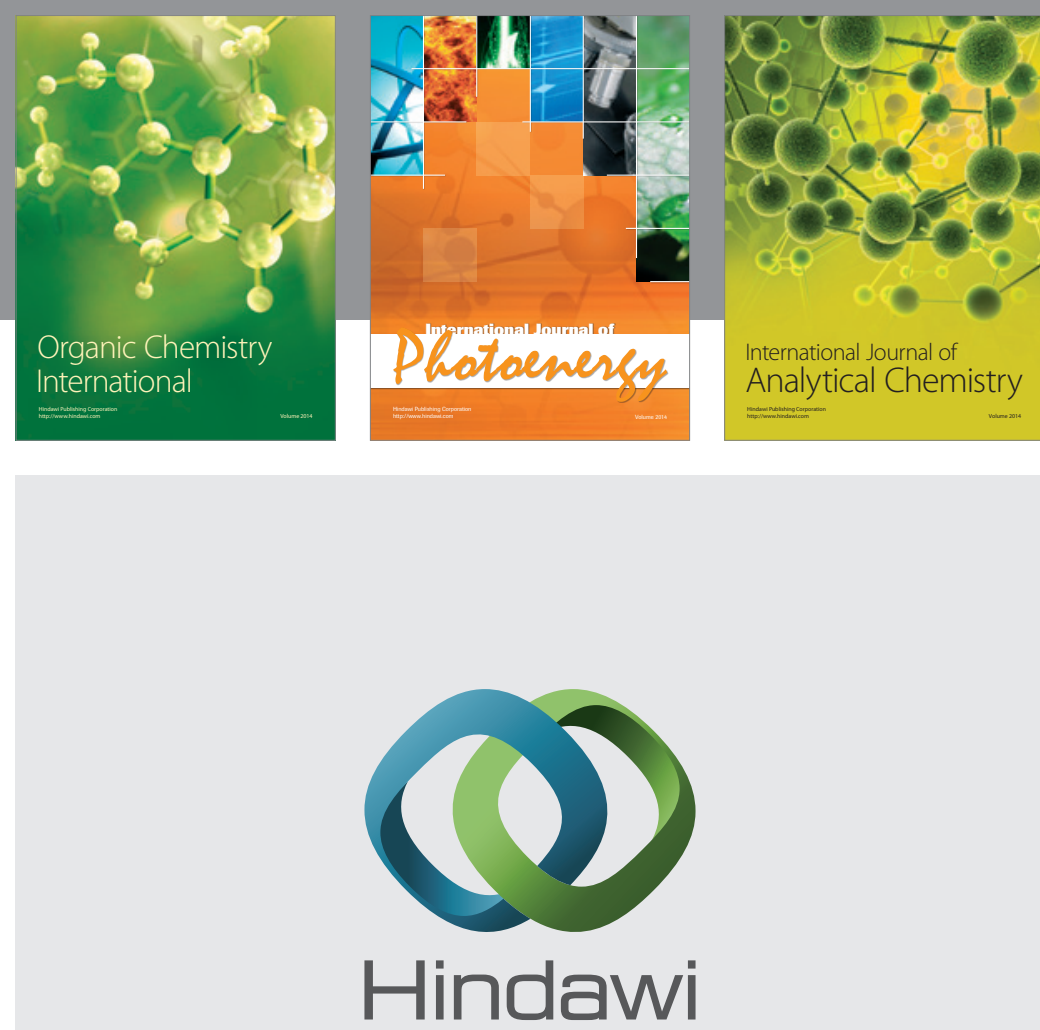

Submit your manuscripts at

http://www.hindawi.com
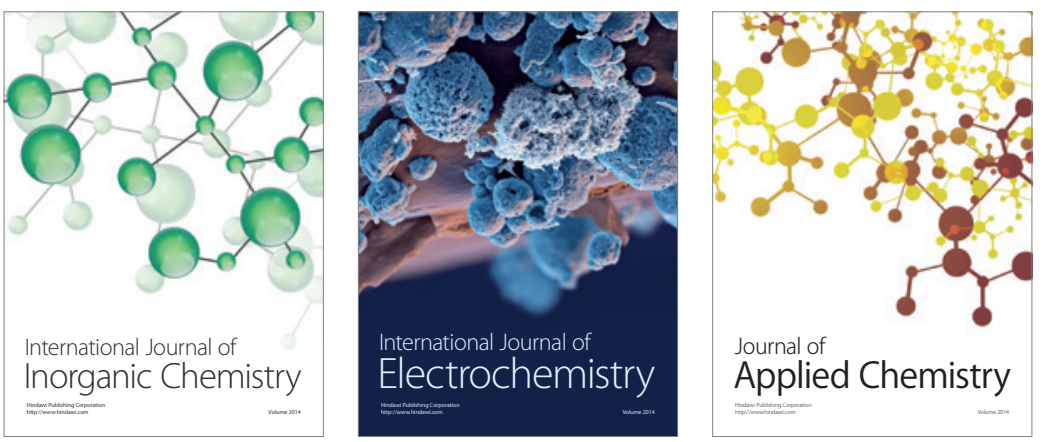

Journal of

Applied Chemistry
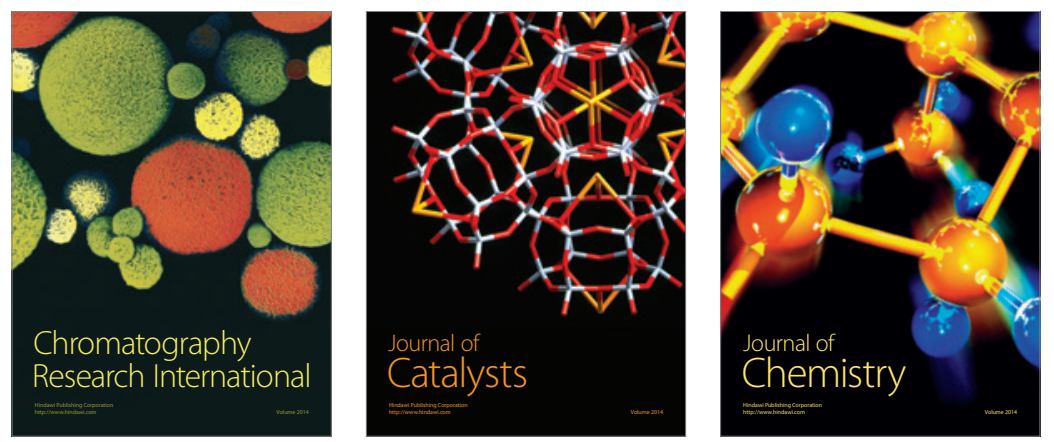
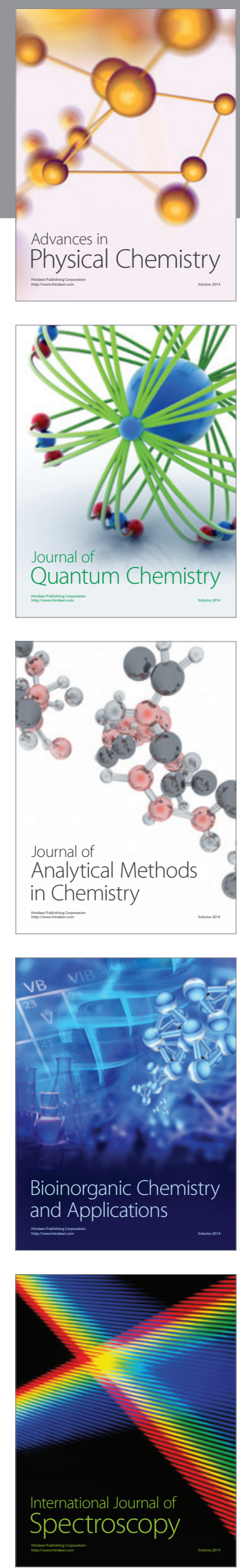